\title{
Role of the Methylene Tetrahydrofolate Reductase (MTHFR) Gene Mutation in Acute Myocardial Infarction
}

\author{
Beáta Jakó, János Sinkó \\ Emergency County Hospital, Miercurea Ciuc, Romania
}

\begin{abstract}
This is a case report of a 36-year-old male who was diagnosed with acute inferior and right ventricular myocardial infarction and treated with percutaneous coronary angioplasty with a drug-eluting stent in the right coronary artery. A profile test for thrombophilia was performed for methylene tetrahydrofolate reductase (MTHFR) gene mutation; the test was positive for a heterozygous mutation - C677C and 1298A. The patient received a long-term treatment with folic acid supplements, taken daily. This case report shows that medical doctors should have an outside-the-box approach for the diagnosis and therapeutic management of young patients who present with acute cardiovascular events. If the patient in question does not present clear cardiovascular risk factors for acute myocardial ischemia, the clinician should seek for possible causes, thus leading to several benefits in the management and secondary prevention of such cases.
\end{abstract}

Keywords: acute myocardial infarction, young patient, MTFR gene mutation, increased homocysteinemia

\section{ARTICLE HISTORY}

Received: 8 January, 2017

Accepted: 17 February, 2017

\section{CORRESPONDENCE}

\section{Beáta Jakó}

Str. Dr. Dénes László nr. 2

530173 Miercurea Ciuc, Romania

Tel: +40 266324193

E-mail: beatajako@gmail.com

\section{INTRODUCTION}

Adjustable risk factors for ischemic heart disease consist of elevated arterial blood pressure, hyperlipidemia, chronic tobacco use, increased body weight, sedentary lifestyle, diabetes mellitus, and anxiety. ${ }^{1}$ Other aspects, such as gender and age, are unchangeable cardiovascular risk factors. ${ }^{2}$ If a patient is admitted to the hospital for typical symptoms of an acute thrombotic event (acute myocardial infarction in the presented case), without any significant cardiovascular risk factors, the clinician is required to exclude genetic disorders as etiology for the acute diagnosis. ${ }^{3}$ Genetic causes that have been shown to be independent risk factors for acute throm- botic events include the mutation of several genes that encode the coagulation factors, such as antithrombin, $\mathrm{C}$ and $\mathrm{S}$ protein, fibrinogen, prothrombin, and factor $\mathrm{V}$ from the clotting cascade. ${ }^{4}$ The genetic mutation of the methylene tetrahydrofolate reductase (MTHFR) gene, which causes a transitional shift in cytosine to thymidine on the 677 nucleotide, leads to a low enzymatic activity and a modified folic acid state with increased folate necessity. The $677 \mathrm{~T}$ mutation of the gene that encodes the MTHFR causes C/C, C/T, and T/T genotypes, leading to an elevated serum level of homocysteine in human subjects. ${ }^{5,6}$ Increased levels of homocysteinemia lead to early atherosclerotic lesion formation and venous thromboembolism. ${ }^{7}$ 

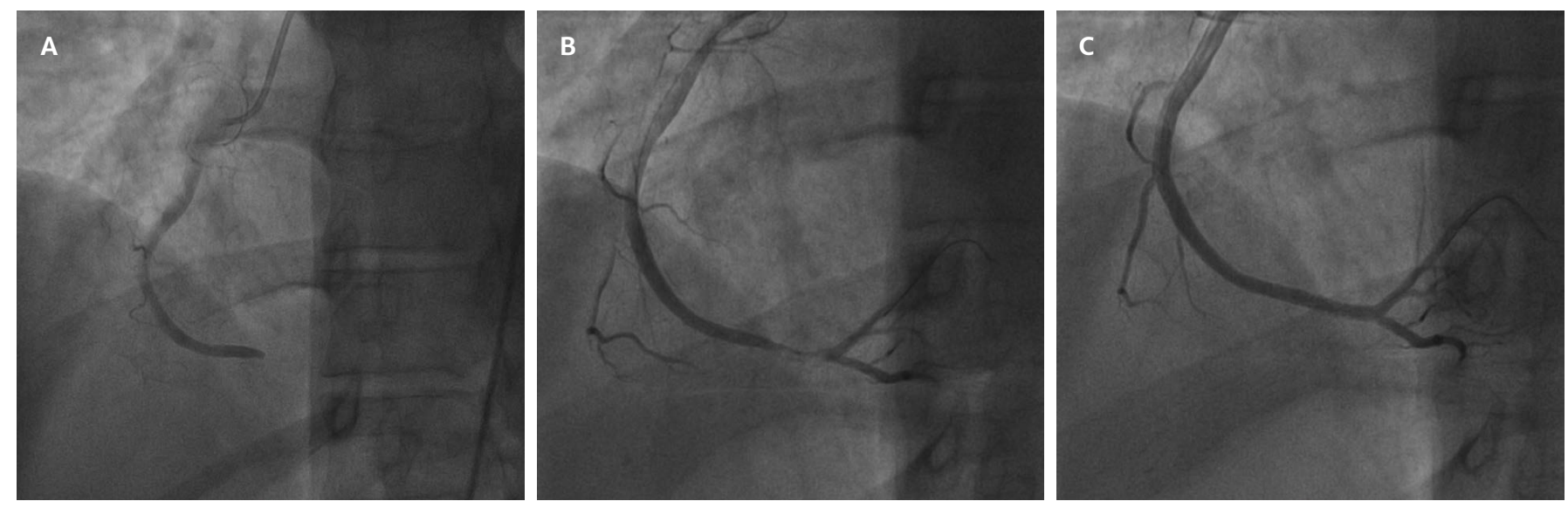

FIGURE 1. Periprocedural coronary angiogram. A - right coronary stenosis, $B$ - after thrombus aspiration, $C$ - postinterventional

This manuscript is a report on a 36-year-old male who was admitted in emergency conditions, with acute chest pain that proved to be an acute right ventricular myocardial infarction (MI). A profile test for thrombophilia was performed for MTHFR gene mutation; the test was positive for a heterozygous mutation $-\mathrm{C} 677 \mathrm{C}$ and $1298 \mathrm{~A}$.

\section{CASE PRESENTATION}

The patient, male, 36 years old, presented to the Emergency Department after 3 hours from the onset of a constrictive, acute chest pain that irradiated down his left arm, with diaphoresis, nausea, and fatigue. The patient's medical history was negative for diabetes, high blood pressure, and chronic tobacco use. The patient did not present any family history of cardiac or cerebrovascular events. The clinical examination on admission revealed a regular heart beat (91/ min) and normal blood pressure (137/71 $\mathrm{mmHg}$ ), with no other pathological modifications. The electrocardiography was illustrative of a postero-inferior and right ventricular ST elevation myocardial infarction (ST-segment elevation in leads D2, D3, aVF, V3R-V5R, ST depression in leads D1, aVL, V1-V2). The patient underwent emergency invasive coronary angiography that showed the acute thrombotic occlusion of the right coronary artery, for which a primary percutaneous coronary angioplasty with a drug-eluting stent was performed (Figure 1). The angiographic aspect of the left coronary artery did not show any atherosclerotic lesions. The laboratory tests were normal for the complete blood cell count, with normal ranges of serum cholesterol triglycerides, kidney and hepatic enzymes.

Following the percutaneous coronary intervention, the patient showed a complete resolution of ST-segment elevation, as well as the disappearance of chest pain and other symptoms. The full hospitalization time was 5 days.
Due to the young age of the subject, screening for thrombophilia was performed, which included testing for MTHFR gene mutation. The screening results showed that the patient was positive for a heterozygous (C677C and 1298A) MTHFR gene mutation. Also, the patient had an increased serum level of homocysteine: $10.5 \mu \mathrm{mol} / \mathrm{L}$ (normal range $<10 \mu \mathrm{mol} / \mathrm{L}$ ).

A hematology consult was requested, which recommended decreasing the homocysteine level, without longterm oral anticoagulant therapy. The patient has been followed-up in out-patient conditions at regular time periods.

The patient agreed to the publication of his data and the institution where the patient had been admitted approved the publication of the case.

\section{DISCUSSION}

Coronary atherosclerotic lesions appear as a result of a complex process that takes place within the vessel wall. Several studies have evaluated the involvement of homocysteine in the process of cell injury at the level of the endothelium. ${ }^{8,9}$

Genetic polymorphism associated with several risk factors leads to increased incidence of ischemic heart disease. Elevated serum levels of homocysteine, caused by MTHFR gene mutation, can be treated with folate supplements. It has been shown that the clinical evolution of arterial or venous thrombotic events in patients with MTHFR gene mutation and increased homocysteine levels improves under supplementation with folic acid, as seen also in this case report. ${ }^{10}$ Gene disorders that affect clotting factor V, prothrombin, and homocysteine, increase the risk of developing acute coronary syndromes or stroke, especially at a young age. ${ }^{11}$ Nevertheless, the screening protocol applies to a relatively low number of subjects, since the number of 
patients who present the association of acute myocardial infarction and these mutations is modest. The tendency for thrombotic events, either arterial or venous, analyzed individually, is influenced by the complex interaction between local and systemic processes. ${ }^{12}$

In the case presented here, screening for MTHFR gene mutation was performed due to the patient's age and lack of cardiovascular risk factors.

We found few cases with acute MI and ivi'Hir $R$ gene mutation in the literature. The first report was published in 1997, on a 35-year-old male patient who presented with acute MI, a homozygous MTHFR gene mutation that led to hyperhomocysteinemia, and a heterozygous mutation of the Leiden $\mathrm{V}$ factor gene. ${ }^{13}$ The patient also presented several risk factors for ischemic heart disease, including late onset type I diabetes and increased cholesterol level. The other described cases also presented a homozygote mutation (with or without other gene mutations), risk factors such as smoking, use of oral contraceptive medication, obesity, and developed myocardial infarction. The particularity of our case is that a heterozygote MTHFR gene mutation was found in a patient with no significant medical history and no cardiovascular risk factors who has been admitted under emergency conditions for acute myocardial infarction.

Establishing an accurate prognosis and predicting future adverse cardiovascular events in patients with MTHFR gene mutation is far from easy, due to its heterogenous mutation process and various forms of clinical presentation. Nevertheless, medical practitioners should have an outside-the-box approach to such patients, especially when young patients present with acute $\mathrm{MI}$ in the lack of any other cardiovascular risk factors.

\section{CONCLUSIONS}

When the presentation diagnosis is not explained by established pathophysiological mechanisms and there are no obvious and identifiable etiological explanations, clinicians should seek for rare causes that will eventually lead to a complex therapeutic approach, beneficial for both the patients and their families.

\section{CONFLICT OF INTEREST}

Nothing to declare.

\section{REFERENCES}

1. Warren T, Barry V, Hooker S, Xuemei S, ChurchT, Blair S. Sedentary Behaviors Increase Risk of Cardiovascular Disease Mortality in Men. Med Sci Sports Exerc. 2010;42:879-885. doi:10.1249/MSS.ob013e3181c3aa7e.

2. Maas A, Appelman Y. Gender differences in coronary heart disease. Neth Heart J. 2010;18:598-602.

3. Kumar A, Cannon C. Acute Coronary Syndromes: Diagnosis and Management, Part I. Mayo Clin Proc. 2009;84:917-938.

4. Previtali E, Bucciarelli P, Passamonti S, Martinelli I. Risk factors for venous and arterial thrombosis. Blood Tranfus. 2011;9:120-138. doi: 10.2450/2010.0066-10.

5. Li XY, Cheng F, Zhang AJ, et al. Folate Deficiency and Gene Polymorphisms of MTHFR, MTR and MTRR Elevate the Hyperhomocysteinemia Risk. Clin Lab. 2017;63:523-533. doi: 10.7754/Clin.Lab.2016.160917.

6. Amrani-Midoun A, Kiando SR, Treard C, Jeunemaitre X, Bouatia-Naji N. The relationship between MTHFR C677T gene polymorphism and essential hypertension in a sample of an Algerian population of Oran city. Int J Cardiol. 2016;225:408411. doi: 10.1016/j.ijcard.2016.10.027.

7. Ganguly P, Alam SA. Role of homocysteine in the development of cardiovascular disease. Nutr J. 2015;14:6. doi: 10.1186/14752891-14-6.

8. Nakai K, Naki K, HabanoW, Gurwitz D. Correlation between C677 T MTHFR gene polymorphism, plasma homocysteine levels and the incidence of CAD. Am J Cardiovasc Drugs. 2001;1:353-361.

9. Zhang W, Wang $M$, Zhang $P$, et al. Role of methylenetetrahydrofolate reductase $677 \mathrm{C} \rightarrow \mathrm{T}$ polymorphism in the development of myocardial infarction: evidence from an original study and updated meta-analysis, Genes Genom. 2016;38:809-817. doi: 10.1007/s13258-016-0424-4.

10. Trabetti E. Homocysteine, MTHFR gene polymorphisms, and cardio-cerebrovascular risk. J Appl Genet. 2008;49:267-82. doi: 10.1007/BF03195624.

11. Ezzat H, Attia F, Mokhtar A, et al. Prevalence of thrombophilic gene polymorphisms (FVL G1691A and MTHFR C677T) in patients with myocardial infarction. Egyptian Journal of Medical Human Genetics, 2014;15:113-123. http://dx.doi. org/10.1016/j.ejmhg.2014.02.001.

12. Liew S, Gupta E. Methylenetetrahydrofolate reductase (MTHFR) C677T polymorphism: Epidemiology, metabolism and the associated diseases, Eur J Med Genet. 2015;58:1-10. doi: 10.1016/j.ejmg.2014.10.004.

13. Ozgul M, Demircelik M, Gunes M, Eryoncu B. Recurrent Acute Coronary Syndromes With Normal Homocystein Level: A Mutation of MTHFR Gene. Gazi medical Journal. 2014;25:3536. doi: http://dx.doi.org/10.12996/gmj.2014.09. 